\title{
Ansprache des Vorsitzenden Jochen Abr. Frowein
}

Spectabilität, meine sehr verehrten Kolleginnen und Kollegen, meine Damen und Herren, es ist eine hohe Ehre und eine große Freude für mich als Vorsitzender unserer Vereinigung, heute nach 75 Jahren die zweite Tagung der Vereinigung der Deutschen Staatsrechtslehrer in Leipzig zu eröffnen. Am 10. März 1925 hatte der Geheime Justizrat Professor Heinrich Triepel die dritte Jahrestagung der Vereinigung hier in Leipzig eröffnet. Ich begrüße ganz besonders die Damen und Herren, die das erste Mal an einer Tagung unserer Vereinigung teilnehmen, und ich begrüße unsere Gäste, die auf Einladung des Vorstandes an dieser Tagung teilnehmen. Ihnen, Spectabilität, darf ich sehr herzlich dafür danken, daß Sie in Vertretung der Leipziger Juristenfakultät gleich einige Worte an uns richten werden. Heinrich Triepel konnte seinerzeit darauf hinweisen, daß er in demselben Raum, in dem die damals kleine Gruppe der Staatsrechtslehrer sich traf, seine Erste Staatsprüfung abgelegt hatte und daß er im nächsten Zimmer sein Rigorosum bestanden hatte. Er würdigte damals die große Zahl der Namen, die mit der Leipziger Juristenfakultät verbunden sind. Uns allen klingen die Namen Otto Mayer, Wilhelm Eduard Albrecht, Carl Friedrich von Gerber, aber über das öffentliche Recht hinaus Windscheid, Binding, Sohm in ganz besonderer Weise. Als jemand, der viel mit der Rechtslage Deutschlands theoretisch und praktisch zu tun hatte, ist es mir eine besondere Freude, die Tagung in der Stadt zu eröffnen, die so wesentlich zu den Veränderungen im Herbst 1989 beigetragen hat. Ich bin ein wenig stolz darauf, daß mit Herrn Kollegen Nolte ein Mitglied unserer Vereinigung hier anwesend ist, der in der schwierigen Umbruchphase fast ein Jahr lang der Leipziger Juristenfakultät bei den Veränderungen voll zur Seite gestanden hat und zwar in der Zeit, als er unter meiner Verantwortung wissenschaftlicher Referent am Max-Planck-Institut in Heidelberg war.

Die Leipziger Tagung 1925 wurde auch mit einer Ansprache des Reichsgerichtspräsidenten Simons eingeleitet. Er meinte damals, es sei ein Unglück, wenn ein selbständiges Reichsverwaltungsgericht neben das Reichsgericht gestellt werden sollte, ein Unglück für das Reichsgericht und ein Unglück für die Einheitlichkeit des deutschen Rechtslebens. In Kürze wird das Bundesverwaltungsgericht - wie Sie wissen von Berlin nach Leipzig umziehen und das Gebäude des alten Reichsgerichts beziehen, was freilich für die Aufgabe zu klein ist und mit er- 
heblichen Anbauten versehen werden muß. Auch hier gibt es also eine Beziehung zu der Tagung vor 75 Jahren, auch wenn die Geschichte anders entschieden hat. Die damals behandelten Themen 1925 betrafen den Schutz des öffentlichen Rechts und die neueste Entwicklung des Gemeindeverfassungsrechts. Es referierten Walter Jellinek, Gerhard Lassar, Fritz Stier-Somlo, Ludwig von Köhler und Hans Helfritz. Das Schicksal von Walter Jellinek und Gerhard Lassar mag uns ganz besonders vor Augen stehen, wenn wir das erste Thema heute nachmittag behandeln, zu dem sich zu meiner großen Freude Herr Dreier und Herr Pauly bereitgefunden haben. Walter Jellinek, Sohn von Georg Jellinek und Enkel eines Rabbiners, verlor seinen Lehrstuhl 1935, konnte aber in Heidelberg bleiben und wurde 1945 sogleich wieder eingestellt. Gerhard Lassar war 1888 in Berlin geboren worden. Er nahm am 1. Weltkrieg vom ersten bis zum letzten Tage als Offizier teil, wurde mit beiden eisernen Kreuzen, dem Hanseatenkreuz und dem Verwundetenabzeichen ausgezeichnet und habilitierte sich 1920 in Berlin bei Triepel. Im Jahre 1925 wurde er Extraordinarius in Hamburg und am 1.1.1934 wurde er wegen seiner Abstammung in den Ruhestand versetzt. Er starb in Hamburg im Januar 1936 mit noch nicht 48 Jahren. Sein Lebenslauf bringt einem das Wort von Ernst-Wolfgang Böckenförde vom Bürgerverrat, der an diesen Menschen begangen worden ist, in Erinnerung. Ich wünsche unserer Tagung einen guten und fruchtbaren Verlauf. 